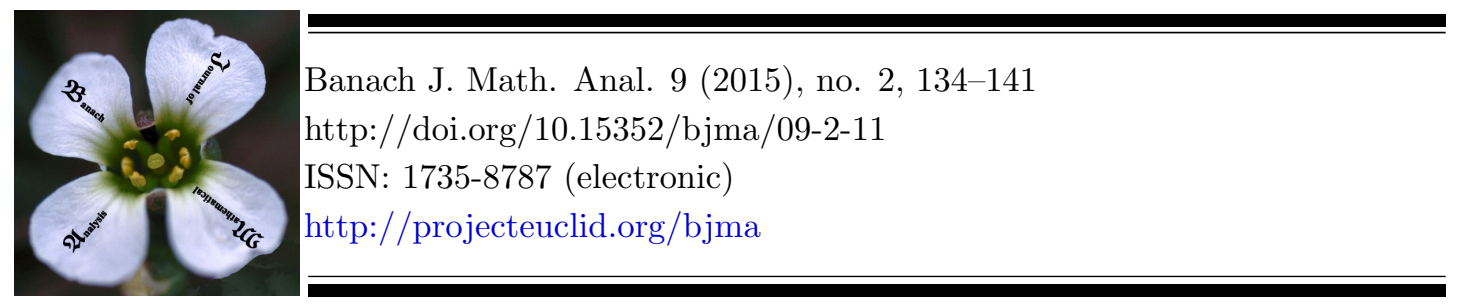

\title{
LINEAR MAPPINGS APPROXIMATELY PRESERVING ORTHOGONALITY IN REAL NORMED SPACES
}

\author{
PAWEŁ WÓJCIK*
}

Communicated by K. Jarosz

\begin{abstract}
In a normed space we introduce an exact and approximate orthogonality relation. We consider classes of linear mappings approximately preserving this kind of orthogonality. We show that, in particular, the property that a linear mapping approximately preserves the $B$-orthogonality is equivalent to that it approximately preserves the $\rho, \rho_{+}$-orthogonality (although these orthogonalities need not be equivalent). Moreover, we show that every approximately orthogonality preserving linear mapping is necessarily a scalar multiple of an almost isometry.
\end{abstract}

\section{INTRODUCTION}

In a normed space, one can define various orthogonality relations and one can consider linear mappings preserving this relations. For example, for the BirkhoffJames orthogonality:

$$
x \perp_{\mathrm{B}} y: \Leftrightarrow \forall_{\lambda \in \mathbb{K}} \quad\|x\| \leqslant\|x+\lambda y\| .
$$

it was proved that a linear mapping preserving this orthogonality must be a scalar multiple of an isometry (see [10], [3]). Similar problem has been analyzed for $\rho_{ \pm}, \rho$-orthogonality in [6],[13] (this notions will be defined in the present section). The paper [5] contains the result about the isosceles orthogonality. In this paper, we give some characterization of linear mappings approximately preserving orthogonality in real normed spaces.

Date: Received: Feb. 22, 2014; Accepted: Jun. 29, 2014.

* Corresponding author.

2010 Mathematics Subject Classification. Primary 46B20; Secondary 46C50, 47B99.

Key words and phrases. Orthogonality in normed spaces, norm derivative, linear mappings orthogonality preserving, approximate orthogonality. 
From this moment, throughout this paper, all normed spaces are assumed to be over reals and at least two-dimensional. Let $(X,\|\cdot\|)$ be a real normed space. We define two mappings $\rho_{+}^{\prime}, \rho_{-}^{\prime}: X \times X \rightarrow \mathbb{R}$ :

$$
\rho_{ \pm}^{\prime}(x, y):=\lim _{t \rightarrow 0^{ \pm}} \frac{\|x+t y\|^{2}-\|x\|^{2}}{2 t}=\|x\| \cdot \lim _{t \rightarrow 0^{ \pm}} \frac{\|x+t y\|-\|x\|}{t} .
$$

This mappings are called norm derivatives. Now, we recall their useful properties (the proofs can be found in [1] and [8]):

$$
\begin{array}{llll}
\text { (nd1) } & \forall_{x, y \in X} & \forall_{\alpha \in \mathbb{R}} & \rho_{ \pm}^{\prime}(x, \alpha x+y)=\alpha\|x\|^{2}+\rho_{ \pm}^{\prime}(x, y) ; \\
\text { (nd2) } & \forall_{x, y \in X} & \forall_{\alpha \geqslant 0} & \rho_{ \pm}^{\prime}(\alpha x, y)=\alpha \rho_{ \pm}^{\prime}(x, y)=\rho_{ \pm}^{\prime}(x, \alpha y) ; \\
\text { (nd2') } & \forall_{x, y \in X} & \forall_{\alpha<0} & \rho_{ \pm}^{\prime}(\alpha x, y)=\alpha \rho_{\mp}^{\prime}(x, y)=\rho_{ \pm}^{\prime}(x, \alpha y) ; \\
\text { (nd3) } & \forall_{x \in X} & \rho_{ \pm}^{\prime}(x, x)=\|x\|^{2} ; \\
\text { (nd4) } & \forall_{x, y \in X} & \left|\rho_{ \pm}^{\prime}(x, y)\right| \leqslant\|x\| \cdot\|y\| .
\end{array}
$$

Moreover, the mappings $\rho_{+}^{\prime}, \rho_{-}^{\prime}$ are continuous with respect to the second variable, but not necessarily with respect to the first one. We have also

$$
\forall_{x, y \in X} \quad \rho_{ \pm}^{\prime}(x, y)=\lim _{t \rightarrow 0} \rho_{ \pm}^{\prime}(x+t y, y) .
$$

The following mapping $\rho^{\prime}: X \times X \rightarrow \mathbb{R}$ was introduced by Miličić [11]:

$$
\rho^{\prime}(x, y):=\frac{1}{2}\left(\rho_{+}^{\prime}(x, y)+\rho_{-}^{\prime}(x, y)\right)
$$

and is called an $M$-semi inner product (briefly $M$-s.i.p.). From the above properties of the mappings $\rho_{+}^{\prime}, \rho_{-}^{\prime}$ we get:

$$
\begin{array}{llll}
\text { (Msip1) } & \forall_{x, y \in X} \quad \forall_{\alpha \in \mathbb{R}} \quad \rho^{\prime}(x, \alpha x+y)=\alpha\|x\|^{2}+\rho^{\prime}(x, y) ; \\
\text { (Msip2) } & \forall_{x, y \in X} \quad \forall_{\alpha \in \mathbb{R}} \quad \rho^{\prime}(\alpha x, y)=\alpha \rho^{\prime}(x, y)=\rho^{\prime}(x, \alpha y) ; \\
\text { (Msip3) } & \forall_{x \in X} \quad \rho^{\prime}(x, x)=\|x\|^{2} ; \\
\text { (Msip4) } & \forall_{x, y \in X} \quad\left|\rho^{\prime}(x, y)\right| \leqslant\|x\| \cdot\|y\| .
\end{array}
$$

Moreover, we have

$$
\forall_{x, y \in X} \quad \rho^{\prime}(x, y)=\lim _{t \rightarrow 0} \rho^{\prime}(x+t y, y) .
$$

We introduce $\rho_{+}$-orthogonality and $\rho_{-}$-orthogonality:

$$
x \perp_{\rho_{+}} y: \Leftrightarrow \rho_{+}^{\prime}(x, y)=0, \quad x \perp_{\rho_{-}} y: \Leftrightarrow \rho_{-}^{\prime}(x, y)=0,
$$

and $\rho$-orthogonality

$$
x \perp_{\rho} y \quad: \Leftrightarrow \quad \rho^{\prime}(x, y)=0 .
$$

Note, that $\perp_{\rho_{+}}, \perp_{\rho_{-}}, \perp_{\rho} \subset \perp_{\mathrm{B}}$. If $(X,\langle\cdot \mid \cdot\rangle)$ is an inner product space, then $\langle y \mid x\rangle=\rho_{+}^{\prime}(x, y)=\rho_{-}^{\prime}(x, y)=\rho^{\prime}(x, y)$ for arbitrary $x, y \in X$. Hence we have $\perp=\perp_{\rho_{+}}=\perp_{\rho_{-}}=\perp_{\rho}=\perp_{\mathrm{B}}$.

In an inner product space an approximate orthogonality ( $\varepsilon$-orthogonality, with $\varepsilon \in[0,1))$ of vectors $x$ and $y$ is naturally defined by:

$$
x \perp^{\varepsilon} y \quad: \Leftrightarrow|\langle x \mid y\rangle| \leqslant \varepsilon\|x\| \cdot\|y\| .
$$

For an approximate Birkhoff orthogonality, we will follow the definition from [4]:

$$
x \perp_{\mathrm{B}}^{\varepsilon} y \quad: \Leftrightarrow \forall \lambda \in \mathbb{R}:\|x\|^{2} \leqslant\|x+\lambda y\|^{2}+2 \varepsilon\|x\| \cdot\|\lambda y\| .
$$

The notions of an approximate $\rho_{ \pm}$and $\rho$-orthogonality were defined in [6] as follows. 


$$
\begin{array}{rlll}
x \perp_{\rho_{+}}^{\varepsilon} y & : \Leftrightarrow \quad\left|\rho_{+}^{\prime}(x, y)\right| \leqslant \varepsilon\|x\| \cdot\|y\| ; \\
x \perp_{\rho_{-}}^{\varepsilon} y & : \Leftrightarrow \quad\left|\rho_{-}^{\prime}(x, y)\right| \leqslant \varepsilon\|x\| \cdot\|y\| ; \\
x \perp_{\rho}^{\varepsilon} y & : \Leftrightarrow \quad\left|\rho^{\prime}(x, y)\right| \leqslant \varepsilon\|x\| \cdot\|y\| .
\end{array}
$$

It is easy to see that $x \perp_{\rho_{+}}^{\varepsilon} y \Rightarrow-x \perp_{\rho_{-}}^{\varepsilon} y$ and $x \perp^{\varepsilon} \rho_{-} y \Rightarrow-x \perp_{\rho_{+}}^{\varepsilon} y$. Moreover, if $x \perp_{\rho_{+}}^{\varepsilon} y$ and $x \perp_{\rho_{-}}^{\varepsilon} y$, then $x \perp_{\rho}^{\varepsilon} y$. Obviously, if the norm in $X$ comes from an inner product, then $\perp_{\rho_{+}}^{\varepsilon}=\perp_{\rho_{-}}^{\varepsilon}=\perp_{\rho}^{\varepsilon}=\perp^{\varepsilon}$ and for $\varepsilon=0$ all the above approximate orthogonalities coincide with the related exact orthogonalities.

The next result (cf. [7]) establishes the connection between $\rho_{ \pm}^{\prime}$ and $\perp_{\mathrm{B}}^{\varepsilon}$.

Theorem 1.1. [7, Theorem 3.1] Let $X$ be a real normed space and let $\varepsilon \in[0,1)$. Then, for arbitrary $x, y \in X$ and $\alpha \in \mathbb{R}$ we have:

$$
x \perp^{\varepsilon}{ }_{\mathrm{B}} y \quad \rho_{-}^{\prime}(x, y)-\varepsilon\|x\| \cdot\|y\| \leqslant 0 \leqslant \rho_{+}^{\prime}(x, y)+\varepsilon\|x\| \cdot\|y\| .
$$

\section{LINEAR MAPPINGS PRESERVING CERTAIN KINDS OF ORTHOGONALITY}

Let $X, Y$ be real normed spaces. We say that a linear mapping $h: X \rightarrow Y$ preserves $\rho_{+}$-orthogonality ( $\rho_{-}$-orthogonality), if $\forall x, y \in X \quad x \perp_{\rho_{+}} y \Rightarrow h x \perp_{\rho_{+}} h y$, $\left(\forall_{x, y \in X} \quad x \perp_{\rho_{-}} y \Rightarrow h x \perp_{\rho_{-}} h y\right)$. Similarly, a linear mapping $f: X \rightarrow Y$ preserves $\rho$-orthogonality if it satisfies:

$$
\forall_{x, y \in X} \quad x \perp_{\rho} y \Rightarrow f x \perp_{\rho} f y .
$$

The following result was proved in [6] and [13].

Theorem 2.1. [6],[13] Let $X, Y$ be real normed spaces, $f: X \rightarrow Y$ a nonzero, linear mapping. Then, the following conditions are equivalent:

(a) $f$ preserves $\rho_{+}$-orthogonality;

(b) $f$ preserves $\rho_{-}$-orthogonality;

(c) $f$ preserves $\rho$-orthogonality;

(d) $\|f x\|=\|f\| \cdot\|x\|, \quad x \in X$;

(e) $\rho_{+}^{\prime}(f x, f y)=\|f\|^{2} \cdot \rho_{+}^{\prime}(x, y), \quad x, y \in X$;

(f) $\rho_{-}^{\prime}(f x, f y)=\|f\|^{2} \cdot \rho_{-}^{\prime}(x, y), \quad x, y \in X$;

(g) $\rho^{\prime}(f x, f y)=\|f\|^{2} \cdot \rho^{\prime}(x, y), \quad x, y \in X$.

In the paper $[6$, Theorem 5] it was proved that $(\mathrm{a}),(\mathrm{b}),(\mathrm{d}),(\mathrm{e}),(\mathrm{f}),(\mathrm{g})$ are mutually equivalent and each of them implies (c). The lacking link was given in [13, Theorems 4.2, 4.3]. Thus in particular, linear mappings preserving $\rho_{+}, \rho_{-}, \rho_{-}$ orthogonality are similarities.

\section{LiNEAR MAPPINGS APPROXIMATELY PRESERVING CERTAIN KINDS OF ORTHOGONALITY}

The class of linear mappings preserving orthogonality can be enlarged by admitting those mappings which only approximately preserve this relation. For inner product spaces $U, W$ one can consider linear mappings $f: U \rightarrow W$ satisfying: for all $x, y \in U, x \perp y \Rightarrow f x \perp^{\varepsilon} f y$.

Let $X, Y$ be real normed spaces. As for $B$-orthogonality, one can consider the class of linear mappings $f: X \rightarrow Y$ satisfying the following condition:

$$
\forall x, y \in X \quad x \perp_{\mathrm{B}} y \Rightarrow f x \perp_{\mathrm{B}}^{\varepsilon} f y .
$$


Similar classes of mappings can be considered for other orthogonality relations. The natural problem is: to describe such a class of approximately orthogonality preserving mappings.

As for $\rho$-orthogonality, one can consider the classes of linear mappings $f: X \rightarrow$ $Y$ satisfying one of the following conditions:

$$
\begin{aligned}
\forall_{x, y \in X} \quad x \perp_{\rho} y & \Rightarrow f x \perp_{\rho}^{\varepsilon} f y ; \\
\forall_{x, y \in X} \quad x \perp_{\rho_{+}} y & \Rightarrow f x \perp_{\rho_{+}}^{\varepsilon} f y ; \\
\forall_{x, y \in X} \quad x \perp_{\rho_{-}} y & \Rightarrow f x \perp_{\rho_{-}}^{\varepsilon} f y .
\end{aligned}
$$

The latter two are equivalent. Indeed, suppose that $f$ approximately preserves $\rho_{+}$-orthogonality and let $x \perp_{\rho_{-}} y$. Thus $-x \perp_{\rho_{+}} y$, hence $-f x \perp_{\rho_{+}}^{\varepsilon} f y$ and finally $f x \perp_{\rho_{-}}^{\varepsilon} f y$, i.e., $f$ approximately preserves $\rho_{-}$-orthogonality. The proof of the reverse is the same. We have proved

Theorem 3.1. Let $X, Y$ be real normed spaces and let $f: X \rightarrow Y$ be linear mapping. Then, the following condition are equivalent:
(a) $f$ satisfies (3.3);
(b) $f$ satisfies (3.4).

Let us quote a result from [7].

Theorem 3.2. [7, Theorem 5.1]. Let $X, Y$ be real normed spaces and let $f: X \rightarrow$ $Y$ be linear and satisfy (3.3) or (3.4). Then $f$ satisfies (3.1).

We will extend this result in the 5th section.

\section{Some Properties OF THE NORM}

A normed space $(X,\|\cdot\|)$ is said to be smooth at the point $x_{o} \in X \backslash\{0\}$, if there is a unique $x^{*} \in X^{*}$ such that $x^{*}\left(x_{o}\right)=\left\|x_{o}\right\|$ and $\left\|x^{*}\right\|=1$. It is known that $X$ is smooth at the point $x_{o} \in X \backslash\{0\}$ if and only if $\rho_{-}^{\prime}\left(x_{o}, y\right)=\rho_{+}^{\prime}\left(x_{o}, y\right)$ for arbitrary $y \in X$ (see [1], [8]). Now, we consider a set

$$
D_{\text {sm }}(X):=\{x \in X: X \text { is smooth at } x\} \cup\{0\} .
$$

The following result (see [1] p. 24 and also [2], [9]) shows that the set $D_{\text {sm }}(X)$ is very large.

Theorem 4.1. Let $(X,\|\cdot\|)$ be a finite-dimensional real normed space. Then there exists a set $F \subset X$ of Lebesgue measure zero such that for all $x$ in $X \backslash F$ and $y$ in $X$ we have $\rho_{+}^{\prime}(x, y)=\rho_{-}^{\prime}(x, y)$, and $X \backslash F$ is dense in $X$.

In this paper, the set $S \subset X$ is called star-shaped, if $\forall_{x \in S} \forall_{\alpha \in \mathbb{R}}: \alpha x \in S$. The following lemma collects the properties of the set $D_{s m}(X)$.

Lemma 4.2. Let $(X,\|\cdot\|)$ be a finite-dimensional real normed space. Then the set $D_{\text {sm }}(X)$ is dense and star-shaped. Moreover, $X \backslash D_{s m}(X)$ is the set of Lebesgue measure zero. 
Proof. Applying Theorem 4.1 we get $X \backslash F \subset D_{\text {sm }}(X)$, thus $D_{s m}(X)$ is dense (since $X \backslash F$ is dense). Next, it is clear that $D_{s m}(X)$ is star-shaped, because $x_{o}$ is a point of smoothness iff $x_{o}$ is a point of smoothness.

Moreover, we have $X \backslash D_{s m}(X) \subset F$ (since $\left.X \backslash F \subset D_{s m}(X)\right)$. Since $F$ is the set of Lebesgue measure zero, we conclude that $X \backslash D_{s m}(X)$ is also the set of Lebesgue measure zero.

\section{MAin RESUlts}

In this section, we give a characterization of linear mappings approximately preserving orthogonality in real normed spaces. Fix $x^{*} \in X^{*}$ and $a \in \mathbb{R}$. The set $M:=\left\{x \in X: x^{*}(x)=a\right\}$ will be called a hyperplane. Let us quote a lemma from [13].

Lemma 5.1. [13, Lemma 4.1] Let $D \subset X$ be a dense, star-shaped subset and let $M$ be a hyperplane such that $0 \notin M$. Then $\overline{M \cap D}=M$.

Turnšek \& Mojškerc [12, Theorem 3.5, Remark 3.1] proved the following result.

Theorem 5.2. [12] Let $X, Y$ be real normed spaces, $\varepsilon \in\left[0, \frac{1}{8}\right)$ and $T: X \rightarrow Y$ a linear mapping satisfying $x \perp_{\mathrm{B}} y \Rightarrow T x \perp_{\mathrm{B}}^{\varepsilon} T y$. Then

$$
\forall_{x \in X} \quad(1-8 \varepsilon)\|T\| \cdot\|x\| \leqslant\|T x\| \leqslant\|T\| \cdot\|x\| .
$$

We will prove that any linear mapping which approximately preserves $\rho$-orthogon -ality, also approximately preserves $\rho_{+}$-orthogonality.

Theorem 5.3. Let $X, Y$ be real normed spaces and let $f: X \rightarrow Y$ be linear and satisfy (3.2). Then $f$ satisfies (3.3).

Proof. For the proof of $(3.2) \Rightarrow(3.3)$ consider the two cases. First, assume that $\operatorname{dim} X=\operatorname{dim} Y=2$. Let $x, y \in X, x \neq 0$. Applying (Msip1) we get $x \perp_{\rho}\left(-\frac{\rho^{\prime}(x, y)}{\|x\|^{2}} x+y\right)$. By (3.2) we have $f x \perp_{\rho}^{\varepsilon}\left(-\frac{\rho^{\prime}(x, y)}{\|x\|^{2}} f x+f y\right)$ and hence

$\left|\rho^{\prime}\left(f x,-\frac{\rho^{\prime}(x, y)}{\|x\|^{2}} f x+f y\right)\right| \leqslant \varepsilon\|f x\| \cdot\left\|-\frac{\rho^{\prime}(x, y)}{\|x\|^{2}} f x+f y\right\|$. Applying again (Msip1) we are able to derive

$$
\forall_{y \in X} \forall_{x \in X \backslash\{0\}}\left|\rho^{\prime}(f x, f y)-\frac{\|f x\|^{2}}{\|x\|^{2}} \rho^{\prime}(x, y)\right| \leqslant \varepsilon\left\|-\frac{\rho^{\prime}(x, y)}{\|x\|^{2}} f x+f y\right\| \cdot\|f x\| .
$$

Next, we show that $f$ is injective. Let $k \in \operatorname{ker} f \backslash\{0\}$. If $x \in X \backslash\{0\}$, then

$$
\left|\rho^{\prime}(f x, f k)-\frac{\|f x\|^{2}}{\|x\|^{2}} \rho^{\prime}(x, k)\right| \leqslant \varepsilon\left\|-\frac{\rho^{\prime}(x, k)}{\|x\|^{2}} f x+f k\right\| \cdot\|f x\|,
$$

Therefore $\left|\frac{\|f x\|^{2}}{\|x\|^{2}} \rho^{\prime}(x, k)\right| \leqslant \varepsilon\left|\frac{\|f x\|^{2}}{\|x\|^{2}} \rho^{\prime}(x, k)\right|$, and hence $\rho^{\prime}(x, k)=0$.

This shows $\forall_{x \notin \operatorname{ker} f} x \perp_{\rho} k$. We recall that $\perp_{\rho} \subset \perp_{\mathrm{B}}$ holds. Therefore, the latter condition becomes

$$
\forall_{x \notin \operatorname{ker} f} x \perp_{\mathrm{B}} k .
$$

Since $\operatorname{dim} X=\operatorname{dim} Y=2$ and $f \neq 0$, $\operatorname{dim} \operatorname{ker} f \leqslant 1$ implies that $X \backslash$ ker $f$ is dense. Thus we can find a sequence $\left(x_{n}\right)_{n=1,2, \ldots}$ such that $x_{n} \notin \operatorname{ker} f$ and $\lim _{n \rightarrow \infty} x_{n}=k$. By 
(5.2) we have $x_{n} \perp_{\mathrm{B}} k$ for $n \in \mathbb{N}$. By continuity of the norm we get $k \perp_{\mathrm{B}} k$, so $k=0$. We proved that $f$ is injective, so $f$ must be surjective, $\operatorname{since} \operatorname{dim} X=\operatorname{dim} Y=2$.

We define the set $S:=D_{s m}(X) \cap f^{-1}\left(D_{s m}(Y)\right)$. It is easy to verify that $S$ is dense and star-shaped. Indeed, the mappings $f, f^{-1}$ are linear. Thus the sets $D_{s m}(X), f^{-1}\left(D_{s m}(Y)\right)$ are star-shaped, so $S$ is also star-shaped. Applying Theorem 4.2 we obtain that $X \backslash D_{s m}(X)$ is the set of Lebesgue measure zero. Moreover, the mappings $f, f^{-1}$ are continuous, so $f$ is a diffeomorphism. Therefore, it is easy to verify that $X \backslash f^{-1}\left(D_{s m}(Y)\right)$ is the set of Lebesgue measure zero because $f\left(Y \backslash D_{s m}(Y)\right)=X \backslash f^{-1}\left(D_{s m}(Y)\right)$. Next, we have

$$
X \backslash S=X \backslash\left(D_{s m}(X) \cap f^{-1}\left(D_{s m}(Y)\right)\right)=\left(X \backslash D_{s m}(X)\right) \cup\left(X \backslash f^{-1}\left(D_{s m}(Y)\right)\right),
$$

so we obtain that $X \backslash S$ is also the set of Lebesgue measure zero. Thus $S$ is dense and star-shaped.

Let $a, b \in X$ be linearly independent. Define $x^{*} \in X^{*}$ by $x^{*}(\alpha a+\beta b):=\alpha$. Then $M:=\left\{z \in X: x^{*}(x)=1\right\}=\{a+t b: t \in \mathbb{R}\}$ is a hyperplane and $0 \notin M$. We have proved that $S$ is dense and star-shaped. Applying Lemma 5.1 we get

$$
\overline{M \cap S}=M \text {. }
$$

Using (5.3) we are able to find a sequence $a+t_{n} b \in S$ such that $\lim _{n \rightarrow \infty} a+t_{n} b=a$, i.e., $\lim _{n \rightarrow \infty} t_{n}=0$. By (5.1) we have

$$
\begin{aligned}
& \left|\rho^{\prime}\left(f\left(a+t_{n} b\right), f b\right)-\frac{\left\|f\left(a+t_{n} b\right)\right\|^{2}}{\left\|a+t_{n} b\right\|^{2}} \rho^{\prime}\left(a+t_{n} b, b\right)\right| \leqslant \\
& \leqslant \varepsilon\left\|-\frac{\rho^{\prime}\left(a+t_{n} b, b\right)}{\left\|a+t_{n} b\right\|^{2}} f\left(a+t_{n} b\right)+f b\right\| \cdot\left\|f\left(a+t_{n} b\right)\right\| .
\end{aligned}
$$

It follows from $a+t_{n} b \in S$ that $a+t_{n} b \in D_{s m}(X)$ and $f\left(a+t_{n} b\right) \in D_{s m}(Y)$. Hence $\rho^{\prime}\left(a+t_{n} b, \cdot\right)=\rho_{+}^{\prime}\left(a+t_{n} b, \cdot\right)$ and $\rho^{\prime}\left(f\left(a+t_{n} b\right), \cdot\right)=\rho_{+}^{\prime}\left(f\left(a+t_{n} b\right), \cdot\right)$. Now the above inequality becomes

$$
\begin{aligned}
& \left|\rho_{+}^{\prime}\left(f\left(a+t_{n} b\right), f b\right)-\frac{\left\|f\left(a+t_{n} b\right)\right\|^{2}}{\left\|a+t_{n} b\right\|^{2}} \rho_{+}^{\prime}\left(a+t_{n} b, b\right)\right| \leqslant \\
& \leqslant \varepsilon\left\|-\frac{\rho_{+}^{\prime}\left(a+t_{n} b, b\right)}{\left\|a+t_{n} b\right\|^{2}} f\left(a+t_{n} b\right)+f b\right\| \cdot\left\|f\left(a+t_{n} b\right)\right\| .
\end{aligned}
$$

Applying (1.1) we get $\left|\rho_{+}^{\prime}(f a, f b)-\frac{\|f a\|^{2}}{\|a\|^{2}} \rho_{+}^{\prime}(a, b)\right| \leqslant \varepsilon\left\|-\frac{\rho_{+}^{\prime}(a, b)}{\|a\|^{2}} f a+f b\right\| \cdot\|f a\|$. We arrived at the following statement:

$$
\left(\begin{array}{c}
a, b \text { are linearly } \\
\text { independent }
\end{array}\right) \Rightarrow\left|\rho_{+}^{\prime}(f a, f b)-\frac{\|f a\|^{2}}{\|a\|^{2}} \rho_{+}^{\prime}(a, b)\right| \leqslant \varepsilon\left\|-\frac{\rho_{+}^{\prime}(a, b)}{\|a\|^{2}} f a+f b\right\| \cdot\|f a\| .
$$

We show that $f$ approximately preserves $\rho_{+}$-orthogonality. Let $x, y \in X$ be such that $x \perp_{\rho_{+}} y$ (we may assume $x \neq 0$ and $y \neq 0$, otherwise (3.3) holds trivially). Thus $\rho_{+}^{\prime}(x, y)=0$. It follows that $x, y$ must be linearly independent. From (5.4) we have $\left|\rho_{+}^{\prime}(f x, f y)\right| \leqslant \varepsilon\|f x\| \cdot\|f y\|$, whence $f x \perp_{\rho_{+}}^{\varepsilon} f y$.

Now, let us prove the general case. Suppose $\operatorname{dim} X \geqslant 2, \operatorname{dim} Y \geqslant 2$. We assume that $x, y \in X \backslash\{0\}$ and $x \perp_{\rho_{+}} y$. Clearly $x, y$ are linearly independent. We 
define $\tilde{f}: \operatorname{Span}\{x, y\} \rightarrow W$ by $\tilde{f}:=\left.f\right|_{\operatorname{Span}\{x, y\}}$, and $W \subset Y$ is a two-dimensional subspace such that $\tilde{f}(\operatorname{Span}\{x, y\}) \subset W$. By the first part, $\tilde{f}$ approximately preserves $\rho_{+}$-orthogonality. We get $\widetilde{f} x \perp_{\rho_{+}}^{\varepsilon} \tilde{f} y$, and hence $f x \perp_{\rho_{+}}^{\varepsilon} f y$.

We will prove that any linear mapping which approximately preserves $B$ orthogonality, also approximately preserves $\rho$-orthogonality.

Theorem 5.4. Let $X, Y$ be real normed spaces and let $f: X \rightarrow Y$ be linear and satisfy (3.1). Then $f$ satisfies (3.2).

Proof. For the proof of $(3.1) \Rightarrow(3.2)$ consider the two cases. First, assume that $\operatorname{dim} X=\operatorname{dim} Y=2$. We define the set $S:=D_{s m}(X) \cap f^{-1}\left(D_{s m}(Y)\right)$. Let $y \in X$, $x \in S, x \neq 0$. Applying (Msip1) we get $x \perp_{\rho}\left(-\frac{\rho^{\prime}(x, y)}{\|x\|^{2}} x+y\right)$. We recall that $\perp_{\rho} \subset \perp_{\mathrm{B}}$ holds. Therefore, the latter condition becomes $x \perp_{\mathrm{B}}\left(-\frac{\rho^{\prime}(x, y)}{\|x\|^{2}} x+y\right)$. By (3.1) we have $f x \perp_{\mathrm{B}}^{\varepsilon}\left(-\frac{\rho^{\prime}(x, y)}{\|x\|^{2}} f x+f y\right)$. Applying Theorem 1.1 we get

$$
\begin{aligned}
& \rho_{-}^{\prime}\left(f x,-\frac{\rho^{\prime}(x, y)}{\|x\|^{2}} f x+f y\right)-\varepsilon\|f x\| \cdot\left\|-\frac{\rho^{\prime}(x, y)}{\|x\|^{2}} f x+f y\right\| \leqslant 0 \leqslant \\
\leqslant & \rho_{+}^{\prime}\left(f x,-\frac{\rho^{\prime}(x, y)}{\|x\|^{2}} f x+f y\right)+\varepsilon\|f x\| \cdot\left\|-\frac{\rho^{\prime}(x, y)}{\|x\|^{2}} f x+f y\right\| .
\end{aligned}
$$

Since $x \in S$, we have $f x \in D_{s m}(Y)$. Thus $\rho_{-}^{\prime}(f x, \cdot)=\rho^{\prime}(f x, \cdot)=\rho_{+}^{\prime}(f x, \cdot)$. Now, we can put $\rho^{\prime}$ in place of $\rho_{+}^{\prime}$ and $\rho_{-}^{\prime}$. Now the above inequalities become

$$
\forall_{y \in X} \forall_{x \in S \backslash\{0\}}\left|\rho^{\prime}\left(f x,-\frac{\rho^{\prime}(x, y)}{\|x\|^{2}} f x+f y\right)\right| \leqslant \varepsilon\left\|-\frac{\rho^{\prime}(x, y)}{\|x\|^{2}} f x+f y\right\| \cdot\|f x\| .
$$

Let $a, b \in X$ be linearly independent. Define $x^{*} \in X^{*}$ by $x^{*}(\alpha a+\beta b):=\alpha$. Then $M:=\left\{z \in X: x^{*}(x)=1\right\}=\{a+t b: t \in \mathbb{R}\}$ is a hyperplane and $0 \notin M$. To finish the proof we argue as in the last part of the previous one. In the suitable part we consider (1.3) instead of (1.1). Now the inequality (5.5) becomes

$$
\left(\begin{array}{c}
a, b \text { are linearly } \\
\text { independent }
\end{array}\right) \Rightarrow\left|\rho^{\prime}(f a, f b)-\frac{\|f a\|^{2}}{\|a\|^{2}} \rho^{\prime}(a, b)\right| \leqslant \varepsilon\left\|-\frac{\rho^{\prime}(a, b)}{\|a\|^{2}} f a+f b\right\| \cdot\|f a\| \text {. }
$$

Now, we can use (5.6). In a similar way as in the proof of Theorem 5.3 we obtain $\forall_{x, y \in X} x \perp_{\rho} y \Rightarrow f x \perp_{\rho}^{\varepsilon} f y$. The case $\operatorname{dim} X \geqslant 2, \operatorname{dim} Y \geqslant 2$ is clear.

Finally, from Theorems 5.2, 3.1, 3.2, 5.3 and 5.4, we obtain the following characterization of linear mappings approximately preserving the orthogonality relations.

Theorem 5.5. Let $X, Y$ be real normed spaces, $f: X \rightarrow Y$ a nonzero, linear mapping. Then, the following conditions are equivalent:

(1) $f$ approximately preserves $\rho_{+}$-orthogonality;

(2) $f$ approximately preserves $\rho_{-}$-orthogonality;

(3) $f$ approximately preserves $\rho$-orthogonality;

(4) $f$ approximately preserves B-orthogonality. 
Moreover, each of the above conditions implies

(5) $\forall_{x \in X} \quad(1-8 \varepsilon)\|f\| \cdot\|x\| \leqslant\|f x\| \leqslant\|f\| \cdot\|x\|$.

Note that, in particular, the property that a linear mapping approximately preserves the $B$-orthogonality is equivalent to that it approximately preserves the $\rho_{+}$-orthogonality. Although $\perp_{\mathrm{B}}^{\varepsilon}$ and $\perp_{\rho}^{\varepsilon}$ need not be equivalent unless we assume the smoothness of the norm (see [7, Theorems 3.3, 3.4]). Thus in particular, every linear mapping approximately preserving $\rho$-orthogonality is necessarily a scalar multiple of an almost isometry.

\section{REFERENCES}

1. C. Alsina, J. Sikorska and M. Santos Tomás, Norm Derivatives and Characterizations of Inner Product Spaces, World Scientific, Hackensack, NJ, 2009.

2. D. Amir, Characterization of Inner Product Spaces, Birkhäuser Verlag, Basel-Boston, 1986.

3. A. Blanco and A. Turnšek, On maps that preserve orthogonality in normed spaces, Proc. Roy. Soc. Edinburgh Sect. A 136 (2006), 709-716.

4. J. Chmieliński, On an $\varepsilon$-Birkhoff orthogonality, J. Inequal. Pure and Appl. Math. 6 (2005), no. 3, Art. 79,7 pp.

5. J. Chmieliński and P. Wójcik, Isosceles-orthogonality preserving property and its stability, Nonlinear Anal. 72 (2010), 1445-1453.

6. J. Chmieliński and P. Wójcik, On a $\rho$-orthogonality, Aequationes Math. 80 (2010), 45-55.

7. J. Chmieliński and P. Wójcik, $\rho$-orthogonality and its preservation - revisited, Recent Developments in Functional Equation and Inequalities, Banach Center Publ., Volume 99, Institute of Mathematic, Polish Academy of Sciences, Warszawa 2013, 17-30.

8. S.S. Dragomir, Semi-Inner Products and Applications, Nova Science Publishers, Inc., Hauppauge, NY, 2004.

9. J.R. Giles, Convex Analysis with Applications in Differentiations of Convex Functions, Pitman, Boston, 1982.

10. A. Koldobsky, Operators preserving orthogonality are isometries, Proc. Roy. Soc. Edinburgh Sect. A 123 (1993), 835-837.

11. P.M. Miličić, Sur la G-orthogonalité dans les espaces normés, Mat. Vesnik 39 (1987), 325334 .

12. B. Mojškerc and A. Turnšek, Mappings approximately preserving orthogonality in normed spaces, Nonlinear Anal. 73 (2010), 3821-3831.

13. P. Wójcik, Linear mappings preserving $\rho$-orthogonality, J. Math. Anal. Appl. 386 (2012), $171-176$.

${ }^{1}$ Institute of Mathematics, Pedagogical University of Cracow, Podchorążych

2, Kraków 30-084, Poland.

E-mail address: pwojcik@up.krakow.pl; pawelwojcikmmm@wp.pl 